\title{
Entanglement spectrum of fermionic bilayer honeycomb lattice: Hofstadter butterfly
}

\author{
Z. Moradi and J. Abouie* \\ Department of Physics, Institute for Advanced Studies in Basic Sciences (IASBS), Zanjan 45137-66731, Iran
}

(Dated: August 2, 2021)

\begin{abstract}
We perform an analytical study of the energy and entanglement spectrum of non-interacting fermionic bilayer honeycomb lattices in the presence of trigonal warping in the energy spectrum, onsite energy difference and uniform magnetic field. Employing single particle correlation functions, we present an explicit form for layer-layer entanglement Hamiltonian whose spectrum is entanglement spectrum. We demonstrate that in the absence of trigonal warping, at zero on-site energy difference exact correspondence is established between entanglement spectrum and energy spectrum of monolayer which means that the entanglement spectrum perfectly reflects the edge state properties of the bilayer. We also show that trigonal warping breaks down such a perfect correspondence, however, in $\Gamma$-K direction in hexagonal Brillouin zone, their behaviors are remarkably the same for particular relevances of hopping parameters. In the presence of an on-site energy difference the symmetry of entanglement spectrum is broken with opening an indirect entanglement gap. We also study the effects of a perpendicular magnetic field on both energy and the entanglement spectrum of the bilayer in the presence of trigonal warping and on-site energy difference. We demonstrate that the entanglement spectrum versus magnetic flux has a self similar fractal structure, known Hofstadter butterfly. Our results also show that the on-site energy difference causes a transition from the Hofstadter butterfly to a tree-like picture.
\end{abstract}

PACS numbers: 73.22.-f, 03.67.Mn, 71.70.Di

\section{INTRODUCTION}

Entanglement is a kind of nonlocal correlations that plays key role in condensed matter physics ${ }^{1}$. The study of entanglement provides new insights into topological states of matter, which can not be characterized using local order parameters. For instance, the topological entanglement entropy of von Neumann entanglement entropy, the most commonly used measurement of entanglement, is directly related to the total quantum dimension of fractional quasiparticles ${ }^{2,3}$. The full entanglement spectrum (ES) provides even more complete information than topological entanglement entropy ${ }^{4}$. The entanglement spectrum of a bipartite system with $A$ and $B$ subsystems, can be characterized by the eigenvalues of the reduced density matrix $\rho_{\text {red }}$. of either one of the two subsystems in the ground state of composite system. The reduced density matrix of a subsystem is obtained by tracing out the other subsystem degrees of freedom. As the eigenvalues of the reduced density matrix are non-negative, one can write $\rho_{\text {red. }}=\frac{1}{Z} e^{-\mathcal{H}}$, where $Z=\operatorname{tr}\left(e^{-\mathcal{H}}\right)$ is a partition function at temperature $T=1$ and $\mathcal{H}$ is the entanglement Hamiltonian whose spectrum is the ES.

The notion of entanglement spectrum has now been applied to many different systems. These include quantum Hall monolayers at fractional filling ${ }^{5-13}$, quantum Hall bilayers at filling factor $\nu=1^{14}$, the Kitaev model ${ }^{15}$, one dimensional quantum spin systems ${ }^{16-25}$, Hofstadter poblem $^{26-28}$, interacting fermions on honeycomb lattice ${ }^{29}$ and bosonic critical system in three dimensions ${ }^{30}$. As a result of these studies, the ES depends on the chosen basis to partition the many body Hilbert space. For systems with a bulk energy gap, the ES obtained from some form of spatial cut contains information reflecting the actual excitation spectrum of the systems at issue. ${ }^{7,8,13,31}$. A large amount of focus of the investigation of ES has been with a partition in various systems like two-leg ladders and various bilayer systems, where the edge comprises the entire remaining subsystem. ${ }^{31-40}$. In this systems, broadly speaking, in the strong interlayer coupling limit, the entanglement Hamiltonian is proportional to the subsystems Hamiltonian. However, this is not the case in general and their relation, depends on the couplings between subsystems. In this paper we consider a model of non-interacting free fermions on a bilayer honeycomb lattice where the presence of interlayer skew hoppings breaks down the mentioned proportionality. Utilizing single particle correlations of one layer, we obtain the entanglement Hamiltonian of the system and present a relation between hopping parameters in which, an exact correspondence is established between the ES of the bilayer and energy spectrum of the monolayer (MES). We show that this correspondence breaks down in the presence of trigonal warping in the band structure of the bilayer. However, similar to the band structure, the ES is symmetric with respect to the zero entanglement as well as the trigonal warping appears on the ES. In the presence of on-site energy difference, in spite of the symmetric energy spectrum of bilayer the entanglement spectrum is completely asymmetric.

In the second part of this paper we study the entanglement spectrum of bilayer honeycomb lattices in the presence of a uniform perpendicular magnetic field. We demonstrate that in the absence of trigonal wrapping in energy spectrum, the ES similar to MES has a self-similar fractal structure, dubbed Hofstadter butterfly. We also 
show that, the presence of an on-site energy difference results in a transition from the so-called Hofstadter butterfly to a tree-like picture.

We have organized the rest of this paper as follows. In Sec. II, we introduce the non-interacting fermionic model on a bilayer honeycomb lattice and obtain the energy spectrum of the model in the presence of interlayer skew hoppings and on-site energy differences. In Sec. III using nonzero single particle correlation functions we build the layer-layer entanglement Hamiltonian. By diagonalizing this Hamiltonian we obtain the entanglement spectrum of the bilayer. In this section we investigate in details the effects of trigonal warping and on-site energy difference on the behavior of single particle correlations and their direct relations with the entanglement spectrum of the system. In Sec. IV we investigate the effects of an external perpendicular magnetic field on the energy band structure and entanglement spectrum of bilayer. Finally in Sec. $\mathrm{V}$ we present our conclusions and outlook.

\section{BILAYER HONEYCOMB LATTICE MODEL}

Bilayer honeycomb lattice consists of two stacked hexagonal monolayers which in turn are made of two sublattices. We denote them as $A$ and $B$ for the lower layer and $A^{\prime}$ and $B^{\prime}$ for the upper layer. In the Bernal stacked bilayer honeycomb lattice (BLH) the $A$ and $B^{\prime}$ sublattices are directly situated above each other as illustrated on the top panel of Fig. 1, however the sites on other two sublattices, B and $A^{\prime}$, don't have a counterpart on the other layer that is directly above or below them.
The tight binding Hamiltonian of the model of noninteracting free fermions on BLH latices is given by ${ }^{41}$ :

$$
\begin{aligned}
H & =-\sum_{\vec{k}}\left[t S_{\vec{k}}\left(a_{\vec{k}}^{\dagger} b_{\vec{k}}+h . c .\right)-\Delta\left(a_{\vec{k}}^{\dagger} a_{\vec{k}}+b_{\vec{k}}^{\dagger} b_{\vec{k}}\right)\right] \\
& -\sum_{\vec{k}}\left[t S_{\vec{k}}\left(a_{\vec{k}}^{\prime \dagger} b_{\vec{k}}^{\prime}+h . c .\right)+\Delta\left(a_{\vec{k}}^{\prime \dagger} a_{\vec{k}}^{\prime}+b_{\vec{k}}^{\prime \dagger} b_{\vec{k}}^{\prime}\right)\right] \\
& +\sum_{\vec{k}}\left[t_{v}\left(b_{\vec{k}}^{\dagger} a_{\vec{k}}^{\prime}+a_{\vec{k}}^{\prime \dagger} b_{\vec{k}}\right)-t_{3}\left(S_{\vec{k}} b_{\vec{k}}^{\prime \dagger} a_{\vec{k}}+S_{\vec{k}}^{*} a_{\vec{k}}^{\dagger} b_{\vec{k}}^{\prime}\right)\right],
\end{aligned}
$$

where the summations run over wave vectors in hexagonal Brillouin zone. $a_{\vec{k}}^{\dagger}\left(a_{\vec{k}}^{\prime \dagger}\right), b_{\vec{k}}^{\dagger}\left(b_{\vec{k}}^{\prime \dagger}\right)$ and $a_{\vec{k}}\left(a_{\vec{k}}^{\prime}\right), b_{\vec{k}}\left(b_{\vec{k}}^{\prime}\right)$ are fermionic creation and annihilation operators with wave vector $\vec{k}$ in lower (upper) layer on sublattices $A\left(A^{\prime}\right)$ and $B\left(B^{\prime}\right)$, respectively. $S_{\vec{k}}=\sum_{\ell=1}^{3} e^{i \vec{k} \cdot \vec{\delta}_{\ell}}$ where $\vec{\delta}_{\ell}$ are the positions of three nearest $B$ sites relative to a given $A$ site, which is written as

$$
\vec{\delta}_{1,2}=\frac{a}{2}(1, \pm \sqrt{3}), \quad \vec{\delta}_{3}=a(-1,0),
$$

with $a$ the distance between two neighboring sites. The on-site energy difference between two layers is denoted by $2 \Delta$ which can be controlled by doping or an external electric field. $t$ denotes the hopping within each layer between sublattices and $t_{v}$ is vertical hopping between two sublattices in different layers, $B$ and $A^{\prime}$. The parameter $t_{3}$ is skew hopping between $A$ and $B^{\prime}$ which leads to trigonal warping in the energy spectrum. All these hoppings are illustrated in Fig. 1.

By diagonalizing the Hamiltonian (1), the energy spectra of the bilayer are readily obtained as $\varepsilon_{\vec{k}}= \pm \varepsilon^{ \pm}$, with

$$
\varepsilon^{ \pm}=\sqrt{\Delta^{2}+\frac{1}{2}\left(t_{v}^{2}+\left(t_{3}^{2}+2 t^{2}\right)\left|S_{\vec{k}}\right|^{2} \pm \sqrt{4 t^{2}\left|S_{\vec{k}}\right|^{2}\left(4 \Delta^{2}+t_{v}^{2}+t_{3}^{2}\left|S_{\vec{k}}\right|^{2}\right)+\left(t_{v}^{2}-t_{3}^{2}\left|S_{\vec{k}}\right|^{2}\right)^{2}+8 t^{2} t_{3} t_{v} \Re\left(S_{\vec{k}}^{3}\right)}\right)}
$$

where $\Re$ denotes real part. The band structure is symmetric with respect to zero energy $\left(\varepsilon_{\mathrm{F}}=0\right)$, and the separation of branches depends on the hopping parameters and on-site energy difference (see Fig. 1). In the absence of on-site energy difference, at zero skew hopping the band structure is rotationally symmetric for an area around a $\mathrm{K}$ point and shows a split into four massive bands, with the two inner ones $\left( \pm \varepsilon^{-}\right)$touching at zero energy and the two outer branches $\left( \pm \varepsilon^{+}\right)$separating from the inner ones by gap $t_{v}$. In the presence of skew hoppings, level crossings between $\varepsilon_{+}$and $\varepsilon_{-}$and between $-\varepsilon_{+}$and $-\varepsilon_{-}$occur in $\Gamma-K$ direction and therefore the low energy physics of the system is captured by the two new inner bands $\pm \varepsilon^{+}$. At the edge of entire Brillouin zone these two branches touch at zero energy at an additional Dirac point where they have linear dispersion (Fig. 1, the bottom panels, inset plot). The appearance of three additional Dirac points around a given $\mathrm{K}$ point due to the skew hoppings is known as trigonal warping which is shown by the contour plots in Fig. 1 .

The level crossings in the band structure are removed by on-site energy difference. The on-site energy difference creates separation $2 \Delta$ between inner branches and opens a band gap at the edge of Brillouin zone.

\section{ENTANGLEMENT SPECTRUM OF BILAYER HONEYCOMB LATTICE}

Before we start the discussions on layer-layer ES of the BLH lattice, it is worthwhile to review briefly some mathematical details concerning the ES of fermionic hopping models on two-leg ladders ${ }^{34,42}$ or bilayer square lattices $^{27}$. In these systems the Hamiltonian of each layer 

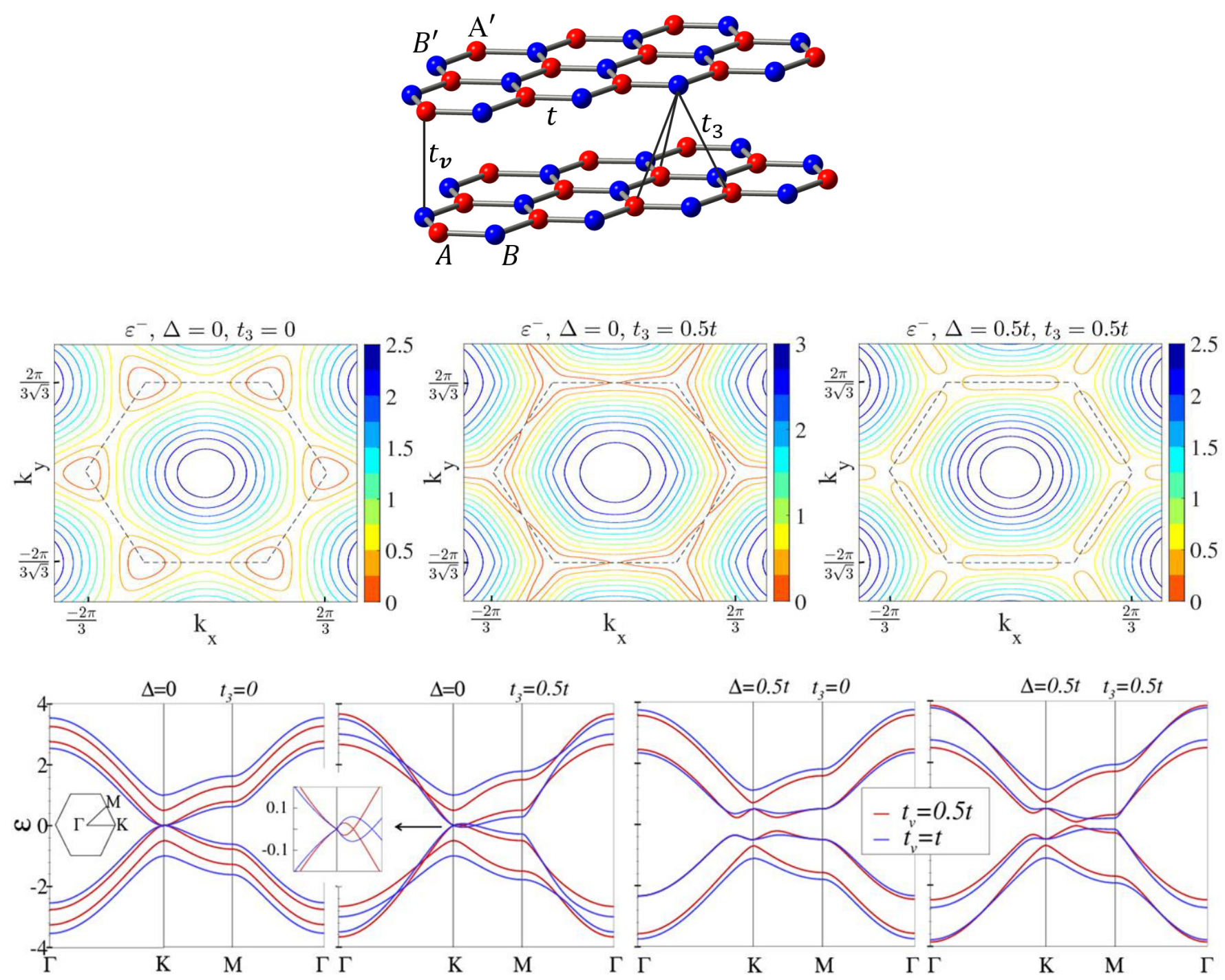

FIG. 1. (Color online) Top: Illustration of Bernal-stacked fermionic bilayer honeycomb lattice with the indication of the within layer and interlayer hopping parameters. Middle: contour plots of energy band $\varepsilon^{-}$for $t_{v}=t$. The dashed lines indicate the edge of the Brillouin zone. Colors show the amount of entanglement. In the presence of interlayer skew hopping three additional Dirac points appear near a given K point. Bottom: the band structure for vertical hopping parameters $t_{v}=0.5 t$ (red lines) and $t_{v}=t$ (blue lines). Two left panels correspond to the energy spectrum in the absence of on-site energy difference for skew hoppings 0 and $0.5 t$. The inset hexagon is the first Brillouin zone with labels of high symmetry points. The enlarged inset plot shows effects of trigonal warping on the band structure in the vicinity of zero energy at the edge of Brillouin zone. Two right panels correspond to energy spectrum in the presence of on-site energy difference, $2 \Delta$.

is generally written in the following diagonal form

$$
H_{\ell}=\sum_{\vec{k}} \varepsilon_{\ell \vec{k}} c_{\ell \vec{k}}^{\dagger} c_{\ell \vec{k}}
$$

where $c_{\ell \vec{k}}^{\dagger}$ and $c_{\ell \vec{k}}$ are fermionic creation and annihilation operators of single particle states with eigenvalues $\varepsilon_{\ell \vec{k}}$. For systems with opposite dispersion in both subsystems i.e., $\varepsilon_{1 \vec{k}}=-\varepsilon_{2 \vec{k}}$, the entanglement Hamiltonian is readily obtained by tracing out subsystem 2 as:

$$
\mathcal{H}_{\text {ent }}=\sum_{\vec{k}} \varepsilon_{\vec{k}}^{e} c_{1 \vec{k}}^{\dagger} c_{1 \vec{k}}
$$

where $\varepsilon_{\vec{k}}^{e}$ denotes the entanglement spectrum which is given by $\varepsilon_{\vec{k}}^{e}=2 \operatorname{arctanh}\left(2\left\langle c_{1 \vec{k}}^{\dagger} c_{1 \vec{k}}\right\rangle\right)$, with the single particle correlation $\left\langle c_{1 \vec{k}}^{\dagger} c_{1 \vec{k}}\right\rangle$ on the ground state of the composite system ${ }^{42-45}$.

The non-interacting free fermions model on BLH lattice (1) is somewhat different from the models considered 

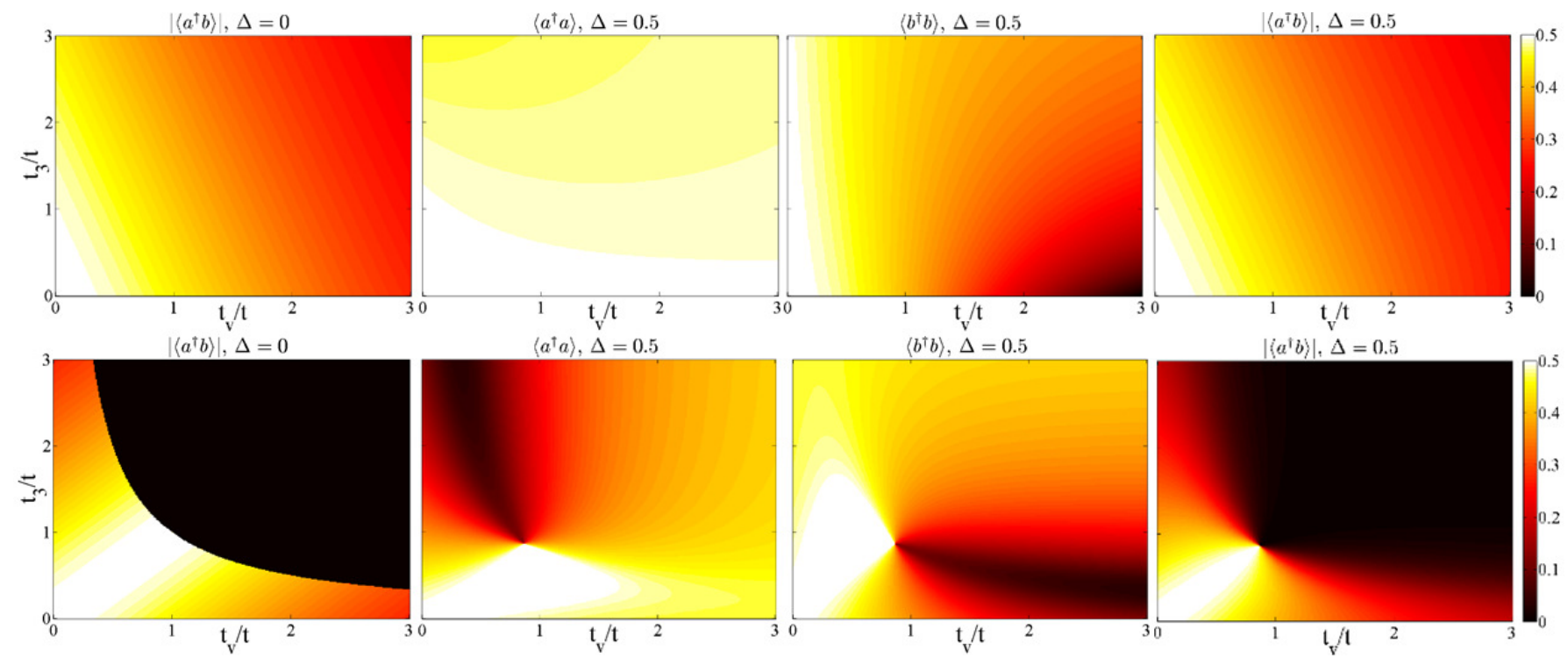

FIG. 2. (Color online) Single particle correlation functions versus scaled hopping parameters $t_{v} / t$ and $t_{3} / t$ in the absence and presence of on-site energy difference. Top: at $\Gamma$ point. Bottom: at $M$ point.

so far, in that the Hamiltonian of layers does not have a diagonal form. We propose the following way for determining the entanglement Hamiltonian of this system. In the first step, we obtain non-zero single particle correlations of lower layer's fermionic operators $(a$ and $b)$ on the ground state of the composite system. In the second step, we build a fictitious entanglement Hamiltonian in terms of the single particle operators with non-zero correlations.

By diagonalizing the Hamiltonian (1) we find out that the single particle correlations:

$$
\left\langle\psi\left|a_{\vec{k}}^{\dagger} a_{\vec{k}}\right| \psi\right\rangle,\left\langle\psi\left|b_{\vec{k}}^{\dagger} b_{\vec{k}}\right| \psi\right\rangle,\left\langle\psi\left|a_{\vec{k}}^{\dagger} b_{\vec{k}}\right| \psi\right\rangle,\left\langle\psi\left|b_{\vec{k}}^{\dagger} a_{\vec{k}}\right| \psi\right\rangle,
$$

are non-zero where $|\psi\rangle$ denotes ground state of composite system. Employing these non-zero correlations we build the entanglement Hamiltonian as:

$$
\mathcal{H}_{\text {ent }}=\sum_{\vec{k}} u_{\vec{k}}^{a} a_{\vec{k}}^{\dagger} a_{\vec{k}}+u_{\vec{k}}^{b} b_{\vec{k}}^{\dagger} b_{\vec{k}}+\left(v_{\vec{k}} a_{\vec{k}}^{\dagger} b_{\vec{k}}+\text { h.c. }\right),
$$

where the unknown $\vec{k}$-dependent coefficients $u_{\vec{k}}^{a}, u_{\vec{k}}^{b}$, and $v_{\vec{k}}$ could be obtained in terms of the nonzero single particle correlation functions (6), however we will see that their explicit forms are not necessary to achieve the ES. By defining

$$
\xi_{\vec{k}}^{ \pm}=\frac{1}{2}\left(u_{\vec{k}}^{a}-u_{\vec{k}}^{b} \pm \sqrt{\left(u_{\vec{k}}^{a}-u_{\vec{k}}^{b}\right)^{2}+4\left|v_{\vec{k}}\right|^{2}}\right),
$$

and employing the following unitary transformation:

$$
\begin{aligned}
& a_{\vec{k}}=\frac{\xi_{\vec{k}}^{-} / v_{\vec{k}}^{*}}{\sqrt{1+\left(\xi_{\vec{k}}^{-} /\left|v_{\vec{k}}\right|\right)^{2}}} \alpha_{\vec{k}}+\frac{\xi_{\vec{k}}^{+} / v_{\vec{k}}^{*}}{\sqrt{1+\left(\xi_{\vec{k}}^{+} /\left|v_{\vec{k}}\right|\right)^{2}}} \beta_{\vec{k}}, \\
& b_{\vec{k}}=\frac{1}{\sqrt{1+\left(\xi_{\vec{k}}^{-} /\left|v_{\vec{k}}\right|\right)^{2}}} \alpha_{\vec{k}}+\frac{1}{\sqrt{1+\left(\xi_{\vec{k}}^{+} /\left|v_{\vec{k}}\right|\right)^{2}}} \beta_{\vec{k}},
\end{aligned}
$$

where, $\alpha_{\vec{k}}$ and $\beta_{\vec{k}}$ are two new fermionic operators, the entanglement Hamiltonian (7) is readily diagonalized as

$$
\mathcal{H}_{\text {ent }}^{d}=\sum_{\vec{k}}\left(\xi_{\vec{k}}^{+} \alpha_{\vec{k}}^{\dagger} \alpha_{\vec{k}}+\xi_{\vec{k}}^{-} \beta_{\vec{k}}^{\dagger} \beta_{\vec{k}}\right) .
$$

Here, $\xi_{\vec{k}}^{ \pm}$are the entanglement spectra which could be written in terms of $n_{\vec{k}}^{+}=\left\langle\alpha_{\vec{k}}^{\dagger} \alpha_{\vec{k}}\right\rangle$ and $n_{\vec{k}}^{-}=\left\langle\beta_{\vec{k}}^{\dagger} \beta_{\vec{k}}\right\rangle$ as

$$
\xi_{\vec{k}}^{ \pm}=2 \operatorname{arctanh}\left(2 n_{\vec{k}}^{ \pm}\right) .
$$

It is now needed to find $n_{\vec{k}}^{ \pm}$on the ground state of the composite system. By substituting $\xi^{ \pm}$from Eq. (8) into the unitary transformations (9) we readily obtain them after simple manipulations in terms of the non zero correlations (6) as follows:

$$
\begin{aligned}
n_{\vec{k}}^{ \pm} & =\frac{1}{2}\left(\left\langle a_{\vec{k}}^{\dagger} a_{\vec{k}}\right\rangle+\left\langle b_{\vec{k}}^{\dagger} b_{\vec{k}}\right\rangle\right. \\
& \left. \pm \sqrt{\left(\left\langle a_{\vec{k}}^{\dagger} a_{\vec{k}}\right\rangle-\left\langle b_{\vec{k}}^{\dagger} b_{\vec{k}}\right\rangle\right)^{2}+4\left|\left\langle a_{\vec{k}}^{\dagger} b_{\vec{k}}\right\rangle\right|^{2}}\right) .
\end{aligned}
$$

In the absence of on-site energy differences, $\left\langle a_{\vec{k}}^{\dagger} a_{\vec{k}}\right\rangle$ and $\left\langle b_{\vec{k}}^{\dagger} b_{\vec{k}}\right\rangle$ are identically equal to $1 / 2$ which implies the existence of the symmetry of single particle distributions on sublattices $A$ and $B$ of lower layer in the ground state of composite system. The single particle correlations $\left|\left\langle a_{\vec{k}}^{\dagger} b_{\vec{k}}\right\rangle\right|$, however, depend on $\vec{k}$ vector as well as the hopping parameters. At a given $\mathrm{K}$ point they are zero, whereas their behavior at other high symmetry points strongly depends upon the hopping parameters. At $\Gamma$ point, for small values of $t_{v} / t$ and $t_{3} / t$ they are almost $1 / 2$ and increasing the interlayer couplings begin to progressively destroy them and for large enough values of 

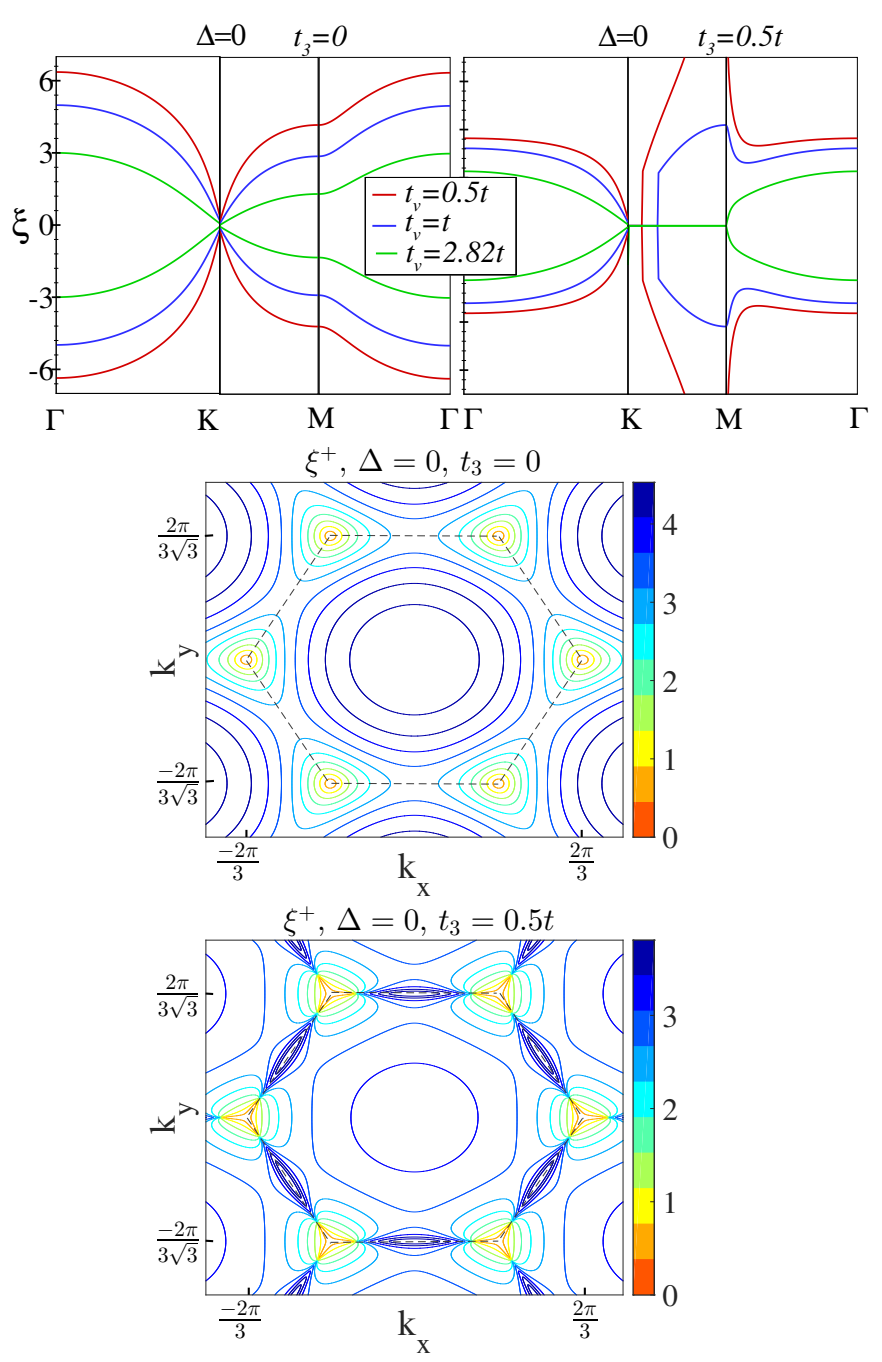

FIG. 3. (Color online) Entanglement spectrum of Bernalstacked fermionic bilayer honeycomb lattice in the absence of on-site energy difference. Top: entanglement spectra versus $\vec{k}$ vector for different values of vertical hopping, in the absence (left panel) and presence (right panel) of interlayer skew hoppings. The green line is both the energy spectrum of a monolayer honeycomb lattice and the ES of bilayer at $t_{v} \cong 2.82 t$. Bottom: contour plots of $\xi_{\vec{k}}^{+}$. The dashed lines indicate the edge of the Brillouin zone. Similar to the energy spectrum of BLH lattice, trigonal warping also appear near $\mathrm{K}$ point in the ES.

interlayer hoppings they will be destroyed (see upper left panel of Fig. 2). At a given M point they fall sharply down to zero by increasing the interlayer couplings (see lower left panel of Fig. 2).

In the absence of on-site energy difference $(\Delta=0)$, the entanglement spectra (11) are simplified to:

$$
\xi_{\vec{k}}^{+}=-\xi_{\vec{k}}^{-}=-2 \operatorname{arctanh}\left(2\left|\left\langle a_{\vec{k}}^{\dagger} b_{\vec{k}}\right\rangle\right|\right)
$$

where, similar to the band structure, they are symmetric with respect to the level with zero entanglement $(\xi=0)$ and their separation depends on the interlayer couplings $t_{v} / t$ and $t_{3} / t$ (see Fig. 3). In the absence of trigonal warping on the energy spectrum of the bilayer, the entanglement spectra are rotationally symmetric for an area around a $\mathrm{K}$ point and shows a split into two massless bands, touching at $\xi=0$. At a particular relevance between interlayer and within layer hoppings, i.e. at $t_{3}=0$ and $t_{v} \cong 2.82 t$, an exact correspondence is established between the entanglement spectra of BLH lattice and monolayer energy spectra which means that the layerlayer ES of BLH lattice reflects the edge state properties of the system, perfectly.

Interlayer skew hoppings considerably affect the ES of the system. As a consequence of trigonal warping some degeneracy and one discontinuity are shown to take place at the edge of entire Brillouin zone, i.e. in K-M direction, due to the level touching at zero energy on band structure of BLH lattice (see Fig. 3, plot $\Delta=0$ and $t_{3}=0.5 t$ ). In the presence of trigonal warping, in general, there is no exact correspondence between entanglement spectra of BLH lattice and the energy spectrum of a monolayer honeycomb lattice, however, in $\Gamma$-K direction for particular relevances between interlayer and within layer hoppings, e.g. for $t_{3} \sim 0.1 t$ and $t_{v} \sim 2.51 t$, the behavior of the ES versus $\vec{k}$ are remarkably similar to the MES.

The on-site energy difference $(\Delta \neq 0)$ causes the correlations $\left\langle a_{\vec{k}}^{\dagger} a_{\vec{k}}\right\rangle$ and $\left\langle b_{\vec{k}}^{\dagger} b_{\vec{k}}\right\rangle$ behave differently with varying interlayer couplings (see Fig. 2) which implies that the on-site energy difference breaks the symmetry of single particle distributions on sublattice $A$ and $B$ of the lower layer. The different behaviors of the single particle correlations, accompany an asymmetry in entanglement spectra with respect to $\xi=0$ (see the upper panel of Fig. $4)$. As a result of this symmetry breaking, the degeneracy of the entanglement spectra at $\xi=0$ is shown to be removed and consequently an indirect entanglement gap (IEG) is opened. Variations of this IEG with increasing the on-site energy difference is shown in lower panel of Fig. 4. In the absence of the trigonal warping it is obviously seen that the IEG increases by $\Delta$, except for weak interlayer vertical hoppings, where the IEG have very small decrease. In the presence of trigonal warping, IEG is found to be increased with a small value of $\Delta$, however more increasing of the on-site energy decreases the IEG progressively and for particular value of interlayer coupling the gap is exactly zero where the two branches overlap indirectly.

\section{HOFSTADTER BILAYER HONEYCOMB LATTICE MODEL}

In this section we investigate the effects of an external uniform magnetic field $\vec{B}=(0,0, B)$ on the entanglement spectrum of the non-interacting free fermions model on the bilayer honeycomb lattice. The effects of a uniform magnetic field, perpendicular to the two dimensional fermionic lattices, could be included by modifying 

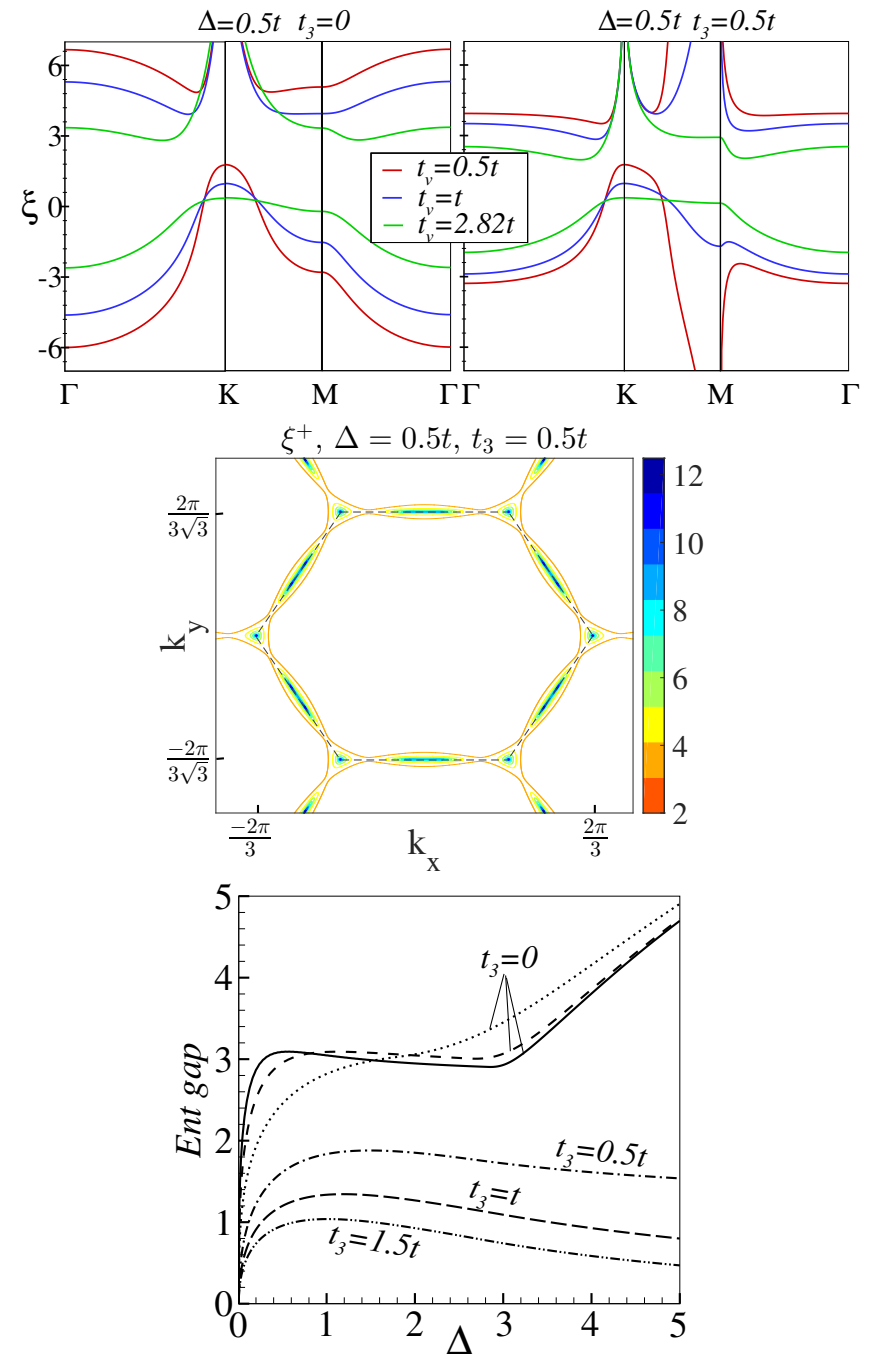

FIG. 4. (Color online) Entanglement spectrum and entanglement gap of Bernal-stacked fermionic bilayer honeycomb lattice in the presence of on-site energy difference. Top: entanglement spectra versus $\vec{k}$ vector for different values of vertical hopping, in the absence (left panel) and presence (right panel) of trigonal warping. Middle: contour plot at the presence of on-site energy difference. Bottom: the indirect entanglement gap versus on-site energy difference. The solid line is for $t_{v}=0.5 t$, the dashed line is for $t_{v}=t$ and the others are for $t_{v}=2.82 t$.

the hopping parameters via the Peierls substitution ${ }^{46}$ as $t_{i j}=t e^{i A_{i j}}$, such that the magnetic flux $\phi$ through each unit cell is a rational multiple $p / q$ of the magnetic flux quantum, $\phi_{0}$. The gauge field $A_{i j}$ can be made periodic by constructing a magnetic super cell of $q$ structural unit cells of the lattice. For a lattice with an $r$ element basis, this results in $q r$ energy sub-bands which in general do not cross. Plotting these energies as a function of $\phi / \phi_{0}=p / q$ yields to a self similar fractal structure, known as Hofstadter butterfly ${ }^{47,48}$.

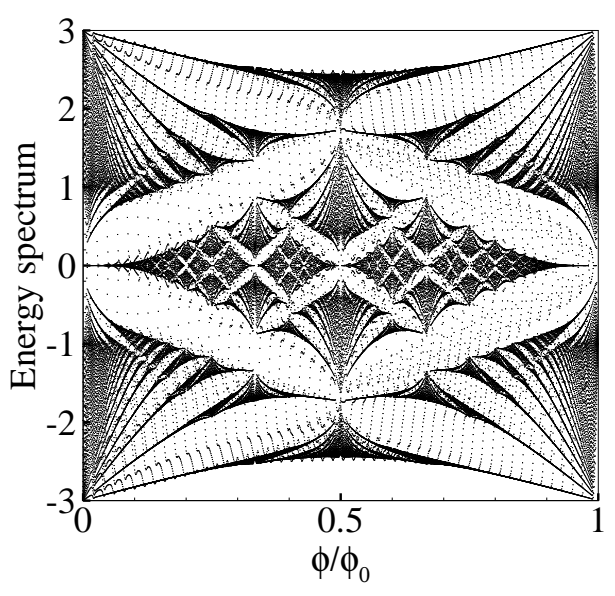

FIG. 5. Energy spectrum of a monolayer honeycomb lattice in the presence of a uniform magnetic field versus reduced magnetic flux $\phi / \phi_{0}=p / q$ where $0 \leq p \leq q=51$.

The actual equations, that need to be solved to obtain the energy spectrum, are known as Harper's equation which is the energy eigenvalue equation for the Hamiltonian matrix $^{49}$. For non-interacting free fermions model on monolayer honeycomb lattice the Harper's equation is equivalent to a $q \times q$ eigenvalue problem. Solving this equation, the energy spectrum versus reduced magnetic flux $\Phi=\phi / \phi_{0}$ has a self similar butterfly structure which is shown in Fig. 5.

For fermionic model on the BLH lattice, Harper's equation is equivalent to the following $4 q \times 4 q$ eigenvalue problem

$$
H_{\vec{k}} \Psi_{\vec{k}}=\varepsilon_{\vec{k}} \Psi_{\vec{k}}
$$

where $\Psi_{\vec{k}}$ is the $4 q$-component spinor

$$
\begin{gathered}
\Psi_{\vec{k}}=\left(\eta_{0 \vec{k}}, \ldots, \eta_{q-1 \vec{k}}, \zeta_{0 \vec{k}}, \ldots, \zeta_{q-1 \vec{k}},\right. \\
\left.\gamma_{0 \vec{k}}, \ldots, \gamma_{q-1 \vec{k}}, \delta_{0 \vec{k}}, \ldots, \delta_{q-1 \vec{k}}\right)^{T},
\end{gathered}
$$

and

$$
H(\vec{k})=\left(\begin{array}{cccc}
\Delta \mathbf{I}_{q \times q} & t h_{\vec{k}} & 0 & t_{3} h_{\vec{k}}^{*} \\
t h_{\vec{k}}^{*} & \Delta \mathbf{I}_{q \times q} & t_{v} \mathbf{I}_{q \times q} & 0 \\
0 & t_{v} \mathbf{I}_{q \times q} & -\Delta \mathbf{I}_{q \times q} & t h_{\vec{k}} \\
t_{3} h_{\vec{k}} & 0 & t h_{\vec{k}}^{*} & -\Delta \mathbf{I}_{q \times q}
\end{array}\right) .
$$

Here, $\mathbf{I}_{q \times q}$ is the $q \times q$ unit matrix and $h_{\vec{k}}$ is the following $q \times q$ matrix

$$
h_{\vec{k}}=\left(\begin{array}{ccccc}
x_{0 \vec{k}} & y_{0 \vec{k}} & 0 & \ldots & z_{\vec{k}} \\
y_{0 \vec{k}}^{*} & x_{1 \vec{k}} & y_{1 \vec{k}} & \ldots & 0 \\
\vdots & \ddots & \ddots & \ddots & \vdots \\
0 & & \ddots & \ddots & y_{q-1 \vec{k}} \\
z_{\vec{k}}^{*} & 0 & \ldots & y_{q-1 \vec{k}}^{*} & x_{q-1 \vec{k}}
\end{array}\right),
$$



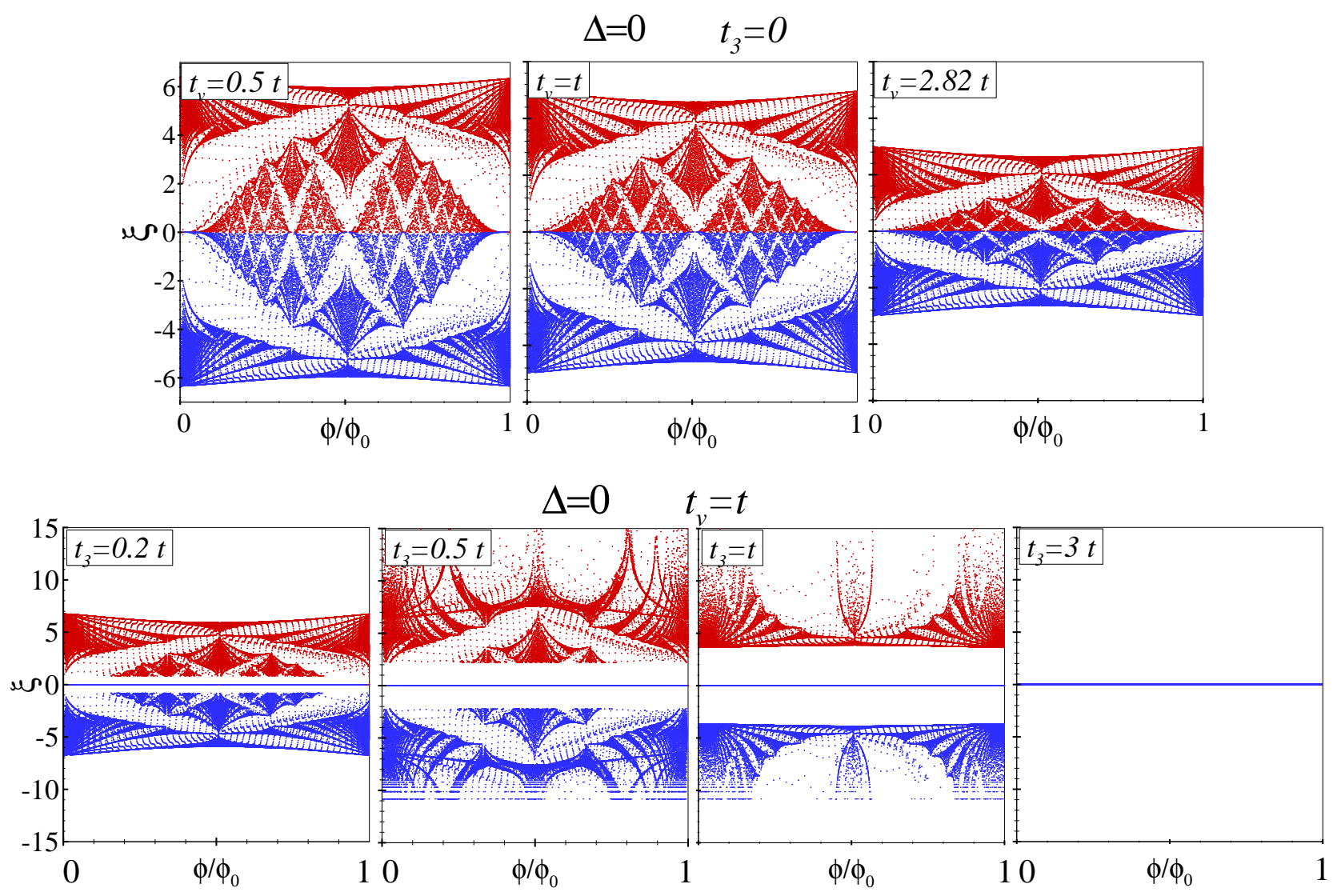

FIG. 6. Entanglement spectrum of BLH lattice versus reduced magnetic flux, in the absence of on-site energy difference, for $0 \leq p \leq q=51$. Top: symmetric self similar structure of entanglement spectrum in the absence of trigonal warping. Bottom: asymmetric spectrum due to the effects of trigonal warping at $t_{v}=t$. For strong interlayer skew hoppings full collapse to zero entanglement is happened.

where,

$$
\begin{aligned}
x_{m \vec{k}} & =2 \cos \left(2 \pi m \Phi+\frac{\sqrt{3}}{2} k_{y}\right), \\
y_{m \vec{k}} & =1+e^{i\left(2 \pi m \Phi+\frac{\sqrt{3}}{2} k_{y}\right)}, \\
z_{\vec{k}} & =e^{-i q k_{x}}+e^{i\left(2 \pi \Phi-\frac{\sqrt{3}}{2} k_{y}-\frac{1}{2} q k_{x}\right)},
\end{aligned}
$$

and $m=0,1, \ldots, q-1$. The diagonalization of the Hamiltonian (15), in general, requires numerics, however explicit analytical results are possible for the special values of the magnetic flux $\phi$. The diagonalization of the Hamiltonian (15) is indeed reduced to the diagonalization of the following $4 \times 4$ Hamiltonian:

$$
H_{m \vec{k}}=\left(\begin{array}{cccc}
\Delta & t \tilde{\varepsilon}_{m \vec{k}} & 0 & t_{3} \tilde{\varepsilon}_{m \vec{k}} \\
t \tilde{\varepsilon}_{m \vec{k}} & \Delta & t_{v} & 0 \\
0 & t_{v} & -\Delta & t \tilde{\varepsilon}_{m \vec{k}} \\
t_{3} \tilde{\varepsilon}_{m \vec{k}} & 0 & t \tilde{\varepsilon}_{m \vec{k}} & -\Delta
\end{array}\right)
$$

where $\tilde{\varepsilon}_{m \vec{k}}$ is $m$-th eigenvalue of $h_{\vec{k}}$.

\section{A. Butterfly entanglement spectrum}

In order to obtain the entanglement spectrum of the non-interacting free fermions model on BLH lattice in the presence of a uniform magnetic field we calculate again the single particle correlations. The non-zero single particle correlations read

$$
\begin{aligned}
& \left\langle\psi_{m}\left|a_{m \vec{k}}^{\dagger} a_{m \vec{k}}\right| \psi_{m}\right\rangle,\left\langle\psi_{m}\left|b_{m \vec{k}}^{\dagger} b_{m \vec{k}}\right| \psi_{m}\right\rangle, \\
& \left\langle\psi_{m}\left|a_{m \vec{k}}^{\dagger} b_{m \vec{k}}\right| \psi_{m}\right\rangle,\left\langle\psi_{m}\left|b_{m \vec{k}}^{\dagger} a_{m \vec{k}}\right| \psi_{m}\right\rangle,
\end{aligned}
$$

where $a_{m \vec{k}}^{\dagger}$ and $b_{m \vec{k}}^{\dagger}\left(a_{m \vec{k}}\right.$ and $\left.b_{m \vec{k}}\right)$ are creation (annihilation) operators of fermions on the lower layer and $\left|\psi_{m}\right\rangle$ is the ground state of $H_{m \vec{k}}$. Using these non-zero correlations one can build the entanglement Hamiltonian of each structural unit cell as

$\mathcal{H}_{m}=\sum_{\vec{k}} u_{m \vec{k}}^{a} a_{m \vec{k}}^{\dagger} a_{m \vec{k}}+u_{m \vec{k}}^{b} b_{m \vec{k}}^{\dagger} b_{m \vec{k}}+\left(v_{m \vec{k}} a_{m \vec{k}}^{\dagger} b_{m \vec{k}}+h . c.\right)$,

where the coefficients $u_{m \vec{k}}^{a}, u_{m \vec{k}}^{b}$, and $v_{m \vec{k}}$ could be obtained in terms of the nonzero single particle correlation 


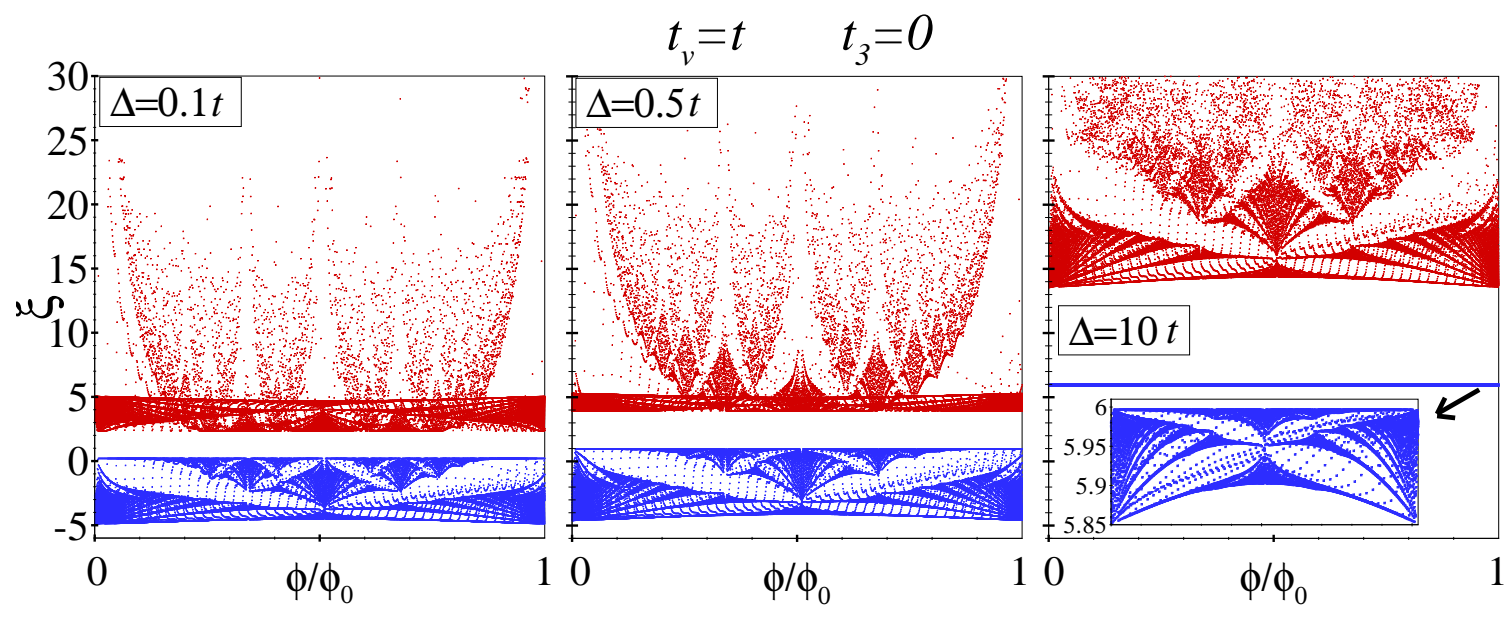

FIG. 7. Self similar structure of the entanglement spectrum of fermionic BLH lattice versus reduced magnetic flux, at $t_{3}=0$, in the presence of on-site energy difference, for $0 \leq p \leq q=51$. A transition from butterfly to a tree-like picture is shown to be occurred for non-zero $\Delta$.

functions (18). By defining

$$
\xi_{m \vec{k}}^{ \pm}=\frac{1}{2}\left(u_{m \vec{k}}^{a}-u_{m \vec{k}}^{b} \pm \sqrt{\left(u_{m \vec{k}}^{a}-u_{m \vec{k}}^{b}\right)^{2}+4\left|v_{m \vec{k}}\right|^{2}}\right)
$$

and using the following unitary transformation:

$$
\begin{aligned}
& a_{m \vec{k}}=\frac{\left(\xi_{m \vec{k}}^{-} / v_{m \vec{k}}^{*}\right) \alpha_{m \vec{k}}}{\sqrt{1+\left(\xi_{m \vec{k}}^{-} /\left|v_{m \vec{k}}\right|\right)^{2}}}+\frac{\left(\xi_{m \vec{k}}^{+} / v_{m \vec{k}}^{*}\right) \beta_{m \vec{k}}}{\sqrt{1+\left(\xi_{m \vec{k}}^{+} /\left|v_{m \vec{k}}\right|\right)^{2}}}, \\
& b_{m \vec{k}}=\frac{\alpha_{m \vec{k}}}{\sqrt{1+\left(\xi_{m \vec{k}}^{-} /\left|v_{m \vec{k}}\right|\right)^{2}}}+\frac{\beta_{m \vec{k}}}{\sqrt{1+\left(\xi_{m \vec{k}}^{+} /\left|v_{m \vec{k}}\right|\right)^{2}}},(21)
\end{aligned}
$$

the Hamiltonian (19) is diagonalized as

$$
\mathcal{H}_{m}^{d}=\sum_{\vec{k}}\left(\xi_{m \vec{k}}^{+} \alpha_{m \vec{k}}^{\dagger} \alpha_{m \vec{k}}+\xi_{m \vec{k}}^{-} \beta_{m \vec{k}}^{\dagger} \beta_{m \vec{k}}\right) .
$$

Here, $\xi_{m \vec{k}}^{ \pm}$are the entanglement spectra which are given by

$$
\xi_{m \vec{k}}^{ \pm}=2 \operatorname{arctanh}\left(2 n_{m \vec{k}}^{ \pm}\right)
$$

where

$$
\begin{aligned}
n_{m \vec{k}}^{ \pm} & =\frac{1}{2}\left(\left\langle a_{m \vec{k}}^{\dagger} a_{m \vec{k}}\right\rangle+\left\langle b_{m \vec{k}}^{\dagger} b_{m \vec{k}}\right\rangle\right. \\
& \left. \pm \sqrt{\left(\left\langle a_{m \vec{k}}^{\dagger} a_{m \vec{k}}\right\rangle-\left\langle b_{m \vec{k}}^{\dagger} b_{m \vec{k}}\right\rangle\right)^{2}+4\left|\left\langle a_{m \vec{k}}^{\dagger} b_{m \vec{k}}\right\rangle\right|^{2}}\right) .
\end{aligned}
$$

In the absence of on-site energy difference, at zero skew hopping the ES versus reduced magnetic flux $\phi / \phi_{0}$, is perfectly symmetric with respect to $\xi=0$, with a self similar structure like Hofstadter butterfly. Increasing interlayer vertical hopping causes $\xi^{+}$and $\xi^{-}$get closer to each other so that at large values of $t_{v}$ they collapse to $\xi=0$. The butterfly-like ES at $t_{v} \cong 2.82 t$ is exactly identical to the MES (compare Fig. 5 and the right plot in upper panels of Fig. 6).
In the presence of trigonal warping the symmetry of butterfly-like ES with respect to $\xi=0$ is shown to be broken (see Fig. 6, the lower panels). For small skew hoppings, the entanglement levels close to $\xi=0$ start to collapse to $\xi=0$ which causes the separation of the two halves of ES. At strong skew hopping, a complete collapse to zero entanglement occurs.

In the presence of on-site energy difference, an asymmetry appears in entanglement spectra with respect to $\xi=0$ and an increase of the entanglement is observed. By increasing $\Delta$ the entanglement levels in the lower half (see Fig. 7, blue levels) get closer to each other, whereas the levels in upper half are shown to be spread forming a self similar tree-like picture. This butterfly-tree transition is due to the symmetry breaking of single particle distributions on sublattice $A$ and $B$ of the lower layer.

\section{CONCLUSIONS AND OUTLOOK}

In this paper we have studied analytically the energy and entanglement spectrum of a non-interacting free fermions model on bilayer honeycomb lattice in the presence of trigonal warping on energy spectrum, onsite energy difference and external magnetic field. The fermionic bilayer honeycomb lattice is somewhat different from the models considered so far in that the Hamiltonian of layers does not have a diagonal form. We have proposed a way for determining the entanglement Hamiltonian of this system by obtaining single particle correlations of one of both layers on the ground state of the composite system. Employing the non-zero single particle correlations we have constructed the entanglement Hamiltonian in terms of these single particle operators. Diagonalizing this Hamiltonian we have found out that in the absence of trigonal warping, at the special value of interlayer vertical coupling $t_{v}=2.82 t$ the entanglement 
spectrum is exactly identical to the edge state energy spectrum. Trigonal warping breaks down this correspondence, however, in $\Gamma-\mathrm{K}$ direction in particular relevances between hopping parameters the entanglement spectrum is remarkably the same as monolayer energy spectrum. Moreover similar to the energy spectrum of the bilayer honeycomb lattice, trigonal warping also exist on the entanglement spectrum. We have also studied the effects of on-site energy difference on the entanglement spectrum of the bilayer and found out that an indirect entanglement gap is opened on ES by on-site energy difference. The behavior of this gap is completely different for bilayers with and without trigonal warping.

In the second part of this paper, we have studied the effects of an external perpendicular magnetic field on the energy and entanglement spectrum of the fermionic bilayer honeycomb lattice. Solving Harper's equation and obtaining the non-zero single particle correlations in the ground state of composite system, we present a non-diagonal entanglement Hamiltonian for Hofstadter bilayer honeycomb lattice. We have shown that the entanglement spectrum versus magnetic flux is perfectly symmetric and, in correspondence with the energy spectrum, possesses the Hofstadter butterfly structure. Finally we have shown that in the presence of an on-site energy difference a transition form butterfly to tree-like picture occurs on entanglement spectrum.

Bilayer graphene is one of the well-known materials with honeycomb lattice which has became the focus of numerous theoretical and experimental works. In bilayer graphene the experimental values ${ }^{50}$ of hopping parameters are $t=3.16 \mathrm{eV}, t_{v}=0.381 \mathrm{eV}$, and $t_{3}=0.38 \mathrm{eV}$. Although they are not in the range where the entanglement spectrum of the bilayer is identical with the energy spectrum of a single layer graphene, similar to the energy spectrum of bilayer graphene the trigonal warping also seen on the entanglement spectrum of the graphene.

Study of the entanglement properties of fermionic bilayer honeycomb lattice with other stacking in the presence of in-plane magnetic field and effects of fermionic interactions are left for the future works.

Note added: During preparing this manuscript we became aware of the paper by Predin, et. al. ${ }^{51}$ where the effects of trigonal warping on the entanglement spectrum of bilayer graphene in the absence of on-site energy difference have been investigated. The authors have found that although the entanglement spectrum shows qualitative geometric differences to the energy spectrum of a graphene monolayer, topological quantities such as Berry-phase-type contributions to Chern numbers agree.
* jahan@iasbs.ac.ir

1 L. Amico, R. Fazio, A. Osterloh, and V. Vedral, Rev. Mod. Pys. 80, 517 (2008).

2 M. Levin and X. G. Wen, Phys. Rev. Lett. 96, 110405 (2006).

3 A. Kitaev and J. Preskill, Phys. Rev. Lett. 96, 110404 (2006).

${ }^{4}$ H. Li and F. D. M. Haldane, Phys. Rev. Lett. 101, 010504 (2008).

5 N. Regnault, B. A. Bernevig, and F. D. M. Haldane, Phys. Rev. Lett. 103, 016801 (2009).

6 O. S. Zozulya, M. Haque, and N. Regnault, Phys. Rev. B 79, 045409 (2009).

7 A. M. Lauchli, E. J. Bergholtz, J. Suorsa, and M. Haque, Phys. Rev. Lett. 104, 156404 (2010).

8 R. Thomale, A. Sterdyniak, N. Regnault, and B. A. Bernevig, Phys. Rev. Lett. 104, 180502 (2010).

9 A. Sterdyniak, N. Regnault, and B. A. Bernevig, Phys. Rev. Lett. 106, 100405 (2011).

${ }^{10}$ R. Thomale, B. Estienne, N. Regnault, and B. A. Bernevig, Phys. Rev. B 84, 045127 (2011).

11 A. Chandran, M. Hermanns, N. Regnault, and B.A. Bernevig, Phys. Rev. B 84, 205136 (2011).

12 A. Sterdyniak, B. A. Bernevig, N. Regnault, and F. D. M. Haldane, New J. Phys. 13, 105001 (2011).

13 X.-L. Qi, H. Katsura, and A. W. W. Ludwig, Phys. Re. Lett. 108, 196402 (2012).

14 J. Schliemann, Phys. Rev. B 83, 115322 (2011).

15 H. Yao and X.-L. Qi, Phys. Rev. Lett. 105, 080501 (2010).

16 P. Calabrese and A. Lefevre, Phys. Rev. A 78, 032329 (2008).

17 L. Fidkowski and A. Kitaev, Phys. Rev. B 81, 134509
(2010)

18 R. Thomale, D. P. Arovas, and B. A. Bernevig, Phys. Rev. Lett. 105, 116805 (2010).

19 F. Pollmann, A. M. Turner, E. Berg, and M. Oshikawa, Phys. Rev. B 81, 064439 (2010).

20 F. Pollmann and J. E. Moore, New J. Phys. 12, 025006 (2010).

21 A. M. Turner, F. Pollmann, and E. Berg, Phys. Rev. B 83, 075102 (2011).

22 G. DeChiara, L. Lepori, M. Lewenstein, and A. Sanpera, Phys. Rev. Lett. 109, 237208 (2012).

${ }^{23}$ L. Lepori, G. De Chiara, and A. Sanpera, Phys. Rev. B 87, 235107 (2013).

${ }^{24}$ W.-J. Rao, X. Wan, and G.-M. Zhang, Phys. Rev. B 90, 075151 (2014).

25 R. Lundgren, J. Blair, P. Laurell, N. Regnault, G. A. Fiete, M. Greiter, and R. Thomale, arXiv:1512.09030

26 Z. Huang and D. P. Arovas, Phys. Rev. B 86, 245109 (2012).

27 J. Schliemann, New. J. Phys. 15, 053017 (2013).

28 J. Schliemann, J. Stat. Mech. P09011 (2014).

29 F. F. Assaad, T. C. Lang, and F. Parisen Toldin, Phys. Rev. B 89, 125121 (2014).

30 Y. Lemonik and A. Mitra, arXiv: 1512.02749

31 R. Lundgren, Y. Fuji, S. Furukawa, and M. Oshikawa, Phys. Rev. B 88, 245137 (2013).

32 D. Poilblanc, Phys. Rev. Lett. 105, 077202 (2010).

33 J. I. Cirac, D. Poilblanc, N. Schuch, and F. Verstraete, Phys. Rev. B 83, 245134 (2011).

${ }^{34}$ I. Peschel and M.-C. Chung, Europhys. Lett. 96, 50006 (2011).

35 A. M. Lauchli and J. Schliemann, Phys. Rev. B 85, 054403 
(2012); J. Schliemann and A. M. Lauchli, J. Stat. Mech. P11021 (2012).

36 S. Tanaka, R. Tamura, and H. Katsura, Phys. Rev. A 86, 032326 (2012).

37 R. Lundgren, V. Chua, and G. A. Fiete, Phys. Rev. B 86, 224422 (2012).

38 X. Chen and E. Fradkin, J. Stat. Mech. P08013 (2013).

39 S. Moudgalya, and F. Pollmann, Phys. Rev. B 91, 155128 (2015).

40 R. A. Santos, C.-M. Jian, and R. Lundgren, arXiv:1511.01489

41 McCann, Edward, and Mikito Koshino, Rep. Prog. Phys. 76, 056503 (2013).

42 I. Peschel, J. Phys. A: Math. Gen. 36, L205 (2003).
43 I. Peschel and V. Eisler, J. Phys. A: Math. Theor. 42, 504003 (2009).

${ }^{44}$ G. Vidal, J. I. Latorre, E. Rico, and A. Kitaev, Phys. Rev. Lett. 90, 227902 (2003).

45 J. I. Latorre and A. Riera, J. Phys. A: Math. Thoer. 42, 504002 (2009).

46 R. E. Peierls, Z. Phys. 80, 763 (1933).

47 M. Y. Azbel, Zh. Eksp. Teor. Fiz. 46, 929 (1964).

48 D. R. Hofstadter, Phys. Rev. B 14, 2239 (1976).

49 Rammal, R., J. Phys. France 46, 1345 (1985).

50 A. B. Kuzmenko, I. Crassee, D. van der Marel, P. Blake and K. S. Novoselov, Phys. Rev. B 80, 165406 (2009).

51 S. Predin, P. Wenk and J. Schliemann, Phys. Rev. B 93, 115106 (2016). 